\title{
"Let's not talk about sex": reconsidering the public health approach to sexually transmissible infections in remote Indigenous populations in Australia
}

\author{
Francis J Bowden and Katherine Fethers
}

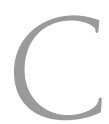
onsider the following: a sight-threatening bacterial infection is endemic in some Indigenous populations in Australia. The disease affects children and adults and requires many years to cause the damage that ultimately leads to blindness in a proportion of those infected. It is associated with poor living conditions, low educational attainment and lack of access to clean water. Australia is the only country outside the developing world where the disease exists. In addition to promoting facial cleanliness and environmental health improvement to reduce the prevalence of this condition, the World Health Organization recommends giving single-dose azithromycin to all children and all adult household contacts of affected people when childhood active trachoma prevalence is $10 \%$ or higher. ${ }^{1}$

Consider another endemic condition in the same population: like the eye disease, it affects young and older people and causes, among other things, cervicitis, urethritis and salpingitis. After several years of infection, individuals are predisposed to ectopic pregnancy and tubal factor infertility. Nearly a quarter of the people in some remote Indigenous communities in Australia are affected. ${ }^{2-4}$ The disease is sexually transmitted, and public health action relies on identifying and treating infected individuals through screening and contact tracing. Public health authorities would consider it unthinkable to offer mass antibiotic treatment to a population affected by a sexually transmitted infection.

We are, of course, comparing the fundamentally different public health approaches that are recommended to control the same organism in the same population - Chlamydia trachomatis in remote Indigenous communities. The major difference is the way we frame the two conditions: one is seen as a disease of poverty, the other is seen to be related to personal and deeply private behaviour.

\section{Sexually transmissible infections and sexual behaviour}

The public health response to sexually transmissible infections (STIs) in any society is usually constructed in the context of sexual behaviour, thus raising, inter alia, the complex issues of morality, privacy, stigma, shame and discrimination. It is self-evident that young people need to receive information about STIs, but a society's prevailing attitudes to sex, the subcultural norms of young people themselves, and the reticence of health professionals can all act as barriers to the implementation of effective strategies. The promotion of condom use and recommendations for the reduction in the rate of partner change are based on the logical, if impractical, premise that STIs can be controlled principally by behaviour modification. While incremental behavioural change may be effective for low-prevalence STIs, endemic disease requires, at least initially, a biomedical intervention.

Insisting that the management of STIs can only be considered in a context of sexual behaviour might be an example of "the excellent being the enemy of the good". Even in affluent societies, there is resistance to the widespread implementation of sexual health promotion. Things are even harder for those with poor

\section{ABSTRACT}

- Sexually transmissible infections (STIs) are hyperendemic in some remote Indigenous populations in Australia.

- Screening programs have had some success in reducing the prevalence of STIs in specific populations, but there has been little overall improvement in the past 10 years.

- We question the usefulness of current practice and urge consideration of a new and radical approach.

- Instead of a "screen, treat and contact trace" strategy, we suggest adopting the same approach as currently accepted for trachoma control: populations reaching a threshold prevalence for a set of marker STIs (identified through sentinel surveillance) should be offered a treatment program aimed at the entire sexually active population.

- We also recommend a parallel program of health promotion and "life skills" education and outline the arguments for such a departure from currently accepted public health policy.

MJA 2008; 188: 182-184

access to education and lower expectations of a long and prosperous life. For those who live in poverty and who suffer societal breakdown, gender inequality and sexual violence, reliance on a health promotional approach alone is problematic, and, it could be argued, dangerously naïve.

\section{STls in Indigenous populations}

STI rates in Indigenous communities are orders of magnitude higher than in non-Indigenous communities. Surveys in remote communities have shown that up to one in four women have an STI (one or more of chlamydia, gonorrhoea, syphilis or trichomoniasis). ${ }^{2-4}$ In some Indigenous populations, STIs are so common that many medical newcomers find it hard to believe that the diagnostic tests they are using are accurate, or worse, that diseases of such endemic prevalence are regarded as "normal" for the group. The limited data that exist demonstrate significant long-term problems of poor health, infertility and ectopic pregnancy. ${ }^{5}$ The acute presentations of STIs in Indigenous populations can also be strikingly different from those seen in urban practice: disseminated gonococcal infection (DGI) is a rare problem in mainstream Australia, but, in Alice Springs, 22 cases of DGI were identified in a 6-month period in 2006 alone. ${ }^{6}$ Pelvic inflammatory disease commonly presents as an acute abdomen in young Indigenous women, and admission to an intensive care unit for complications of the sepsis is not a rare event.

\section{Screening for STls}

In the 1990s, the National Indigenous Australians' Sexual Health Strategy dramatically changed the approach to STI control in 
remote communities by promoting screening and by funding the necessary technologies. ${ }^{7}$ Subsequent work by Nganampa Health in Central Australia showed that screening worked, was acceptable to the affected population and led to sustainable reductions in disease prevalence. ${ }^{8}$ However, more recently there have been anecdotal reports of an increase in the prevalence of disease in some communities, despite repeated screening. Unfortunately, the failure of specific programs has in some quarters been interpreted as representing a fundamental flaw in the whole concept of screening, rather than a predictable consequence of inadequately resourced programs. Infectious disease modelling suggests that, in the absence of behavioural change, the rises are inevitable unless more than $50 \%$ of the population is screened and the screening interval is reduced to less than 12 months. ${ }^{9}$ This is not possible in remote communities with the current level of staffing and health infrastructure. Furthermore, there is an inevitable delay between testing and the return of results: in some cases this is a few days, in others, weeks. The mobility of remote populations can make it difficult to locate screened individuals who have returned positive results. The identification of infected individuals through rapid testing with immediate treatment may help, but current point-of-care tests are not as accurate as laboratory-based tests. ${ }^{10}$ In fact, investigations add only a little to the diagnostic certainty of the clinician if the disease is endemic. (This contrasts with low-prevalence populations, in which testing is the principal guide to the need for treatment.) If a disease affects one in four people in a population, then the positive predictive value of just being a member of that population is $25 \%$. Many screening tests used in low-prevalence populations do not have a positive predictive value this high.

Most STI strategies target specific age groups. In mainstream Australia, the vast majority of chlamydial infections, for example, occur in the 15-30-year-old age group, and it is assumed that "assortative" mixing occurs (ie, people having sex with each other are of roughly the same age). However, although age-specific data in Indigenous populations show that the risk of disease is highest in younger groups, ${ }^{2}$ a greater proportion of the older population is also infected, and anecdotal evidence suggests that "dis-assortative" partner selection is common (eg, a man in his thirties or older often has a partner in her teens or twenties). Furthermore, it is important to recognise other barriers to participation in screening in closed communities - asking people to self-select only if they have had a recent partner change inhibits screening, as individuals who present are potentially identifying themselves as non-monogamous.

\section{A new approach to STI control}

We believe that it is time to radically change the approach to the control of STIs in high-prevalence populations in Australia through the introduction of men's and women's treatment programs. The new programs would involve community-based empirical treatment determined by an assessment of risk at a population level, rather than at the level of the individual. In line with the approach to trachoma control, communities with an STI prevalence of over $10 \%$ would be allocated to a whole-of-population approach, in which all individuals within a particular age range would be offered antibiotic treatment without recourse to an individual risk assessment and without waiting for the results of diagnostic testing. It is known that STIs occur in children aged 10-14 years in some remote communities and that, in the over 30 years age group, there is a higher prevalence of STIs in Indigenous people than in the nonIndigenous population. Therefore, it would be reasonable to pro- pose that everyone over the age of 10 years in Indigenous communities be included. Communities with an STI prevalence of less than $10 \%$ would follow the usual "screen and treat" protocol. The prevalence of STIs in specific populations would be determined through sentinel screening — for example, regular clinic-based testing of a sample of asymptomatic men and women. (The small numbers of patients in many communities could make these data difficult to interpret in some circumstances).

The most prevalent STIs in Indigenous communities (chlamydia, gonorrhoea and trichomoniasis) could be treated with a fourdrug combination. At present, the resistance patterns for gonorrhoea in remote Australia would allow the use of a single oral dose of amoxycillin and probenecid for treatment. Single-dose oral azithromycin and tinidazole could be used to treat chlamydia and trichomoniasis, respectively. Resistance in C. trachomatis is rare, but the effect that these antibiotics have on the resistance patterns of other organisms (such as Streptococcus pneumoniae and S. pyogenes) would need to be closely monitored. To avoid gastrointestinal problems, the four-drug combination might need to be staggered over 24-48 hours.

This proposal is in keeping with WHO practice for other endemic diseases such as river blindness, ${ }^{11}$ trachoma ${ }^{1}$ and schistosomiasis. ${ }^{12}$ It has local analogues in the routine use of antihelminthics in Indigenous children and in community-based scabies treatments. ${ }^{13}$ It acknowledges that individuals are autonomous and amenable to behavioural change. All treatment would be contingent on informed consent from each individual.

We know that the use of azithromycin for trachoma control is acceptable, ${ }^{14}$ but can this strategy work for STI control? A serendipitous finding in Central Australia has demonstrated the potential benefit of the whole-of-population approach. One community underwent a trachoma control program in which azithromycin treatment was given to 264 adults and 100 children between the ages of 5 and 49 years. A routine STI control activity, which followed 6 weeks later, found a substantial difference in the rates of chlamydia and gonorrhoea in individuals who had received azithromycin compared with those who had not $(6.6 \% \mathrm{v}$ $27.4 \%$ ). The overall prevalence of STIs in this community was also lower than that found in other communities in the region. ${ }^{15}$ It is unknown how long this effect is sustained, but the figures indicate a substantial short-term benefit.

The mobility of Indigenous people in remote areas is considered to be one reason for the lack of sustainability of reductions in trachoma prevalence, ${ }^{14}$ and our approach, which avoids a "screen, recall, then treat" sequence, may allow a broader, coordinated regional intervention to be undertaken. People visiting other communities and towns could be included in the programs, and the issue of non-Aboriginal people living in the relevant communities, who may also be at risk of STIs, would need to be considered in designing inclusion criteria for screening.

Concurrently with health service delivery, this model would need to address the issues of sexual violence, sexual exploitation and informal sex-work that are also endemic in many communities. Education programs dealing with life skills and encompassing a wide range of health behaviours would need to be run by a separate team in parallel with, but not necessarily hand-in-hand with, the STI control activities. Remote-area nurses and other medical staff vicariously (and occasionally directly) experience the trauma of physical and sexual assaults, and we believe that they will welcome 


\section{FOR DEBATE}

a properly resourced approach that attempts to deal with both the biomedical and the social issues in a coordinated way.

\section{Conclusions}

The recent national emergency response to protect Aboriginal children in the Northern Territory demonstrates that there is a willingness on the part of the federal government to adequately resource interventions and that access to good clinical services is a necessary first step towards better health. However, we stress that it is impossible to achieve any lasting improvements in the wellbeing of Indigenous people if the deeper social determinants of disease are ignored. The amount of funding and commitment required from the broader Australian community to achieve this is substantial, and requires true cooperation and consultation in addition to strong local and national leadership.

Any new approach to STIs must be carefully introduced, with widespread consultation and agreement obtained from the communities themselves, local health services, the federal government and state and territory health departments before proceeding.

The details of how exactly to deliver the men's and women's programs will require much debate and consideration. But, in light of hyperendemic levels of STIs and the ever-present threat of an HIV epidemic, how can we not explore new ways of preventing and controlling these conditions in the Indigenous population?

\section{Competing interests}

None identified.

\section{Author details}

Francis J Bowden, FRACP, FAChSHM, MD, Professor of Medicine ${ }^{1}$ Katherine Fethers, MB BS, FAChSHM, MMed(STD/HIV), Sexual Health Physician $^{2}$

1 Academic Unit of Internal Medicine, Australian National University Medical School, Canberra, ACT.

2 Melbourne Sexual Health Centre, Melbourne, VIC.

Correspondence: frank.bowden@act.gov.au

\section{References}

1 World Health Organization. Report of the second global scientific meeting on trachoma, 25-27 August 2003. Geneva: WHO, 2003. (WHO/ PBD/GET 03.1.)
2 Bowden FJ, Paterson BA, Mein J, et al. Estimating the prevalence of Trichomonas vaginalis, Chlamydia trachomatis, Neisseria gonorrhoeae, and human papillomavirus infection in indigenous women in northern Australia. Sex Transm Infect 1999; 75: 431-434.

3 Miller GC, McDermott R, McCulloch B, et al. Predictors of the prevalence of bacterial STI among young disadvantaged Indigenous people in north Queensland, Australia. Sex Transm Infect 2003; 79: 332-335.

4 Panaretto KS, Lee HM, Mitchell MR, et al. Prevalence of sexually transmitted infections in pregnant urban Aboriginal and Torres Strait Islander women in northern Australia. Aust N Z J Obstet Gynaecol 2006; 46: 217-224. (doi: 10.1111/j.1479-828X.2006.00577.x.)

5 Kildea S, Bowden FJ. Reproductive health, infertility and sexually transmitted infections in indigenous women in a remote community in the Northern Territory. Aust N Z J Public Health 2000; 24: 382-386.

6 Fethers KL. A disseminated gonococcal infection in Alice Springs [abstract]. Australasian Sexual Health Conference; 2006 Oct 9-11; Melbourne.

7 Commonwealth of Australia. National Indigenous Australians' Sexual Health Strategy 1996-97 to 1998-99: a report of the ANCARD Working Party on Indigenous Australians' Sexual Health. Canberra: Commonwealth of Australia, 1997. http://www.health.gov.au/internet/wcms/publishing.nsf/Content/63F562FEE9FCAB7ECA25722B00834275/\$File/ ancard.pdf (accessed Nov 2007)

8 Miller PJ, Torzillo PJ, Hateley W. Impact of improved diagnosis and treatment on prevalence of gonorrhoea and chlamydial infection in remote Aboriginal communities on Anangu Pitjantjatjara Lands. Med J Aust 1999; 170: 429-432.

9 Bowden FJ, Garnett GP. Trichomonas vaginalis epidemiology: parameterising and analysing a model of treatment interventions. Sex Transm Infect 2000; 76: 248-256.

10 Peeling RW, Holmes KK, Mabey D, Ronald A. Rapid tests for sexually transmitted infections (STIs): the way forward. Sex Transm Infect 2006; 82 Suppl 5: v1-v6.

11 Boatin BA, Richards FO Jr. Control of onchocerciasis. Adv Parasitol 2006; 61: 349-394.

12 Fenwick A, Webster JP. Schistosomiasis: challenges for control, treatment and drug resistance. Curr Opin Infect Dis 2006; 19: 577-582.

13 Northern Territory Department of Health and Community Services. Guidelines for community control of scabies, skin sores and crusted scabies in the Northern Territory. Darwin: NT Government, 2003. http:// www.nt.gov.au/health/cdc/treatment_protocol/scabies.pdf (accessed Dec 2007)

14 Ewald DP, Hall GV, Franks CC. An evaluation of a SAFE-style trachoma control program in Central Australia. Med J Aust 2003; 178: 65-68.

15 Latif A. Effect of community-based trachoma mass treatment on chlamydia and/or gonorrhoea rates [abstract]. Australasian Sexual Health Conference; 2006 Oct 9-11; Melbourne.

(Received 13 Jun 2007, accepted 2 Oct 2007) 